\title{
A Modified Equivalence Principle for Open Surfaces
}

\author{
Manouchehr Takrimi and Levent Gürel \\ Bilkent University Computational Electromagnetics Research Center (BiLCEM), Ankara, TR-06800, Turkey \\ lgurel@gmail.com
}

\begin{abstract}
We introduce a new method for expanding equivalent surface current densities over open surfaces. The new method is based on the equivalence principle, which is theoretically used with closed surfaces. Utilizing low-order basis functions to compute the expansion coefficients of equivalent surface currents and then weighting these coefficients with an appropriate window function compensates for computational field errors originating from using truncated currents at the open boundaries. These modified coefficients can be used to reproduce the original electromagnetic fields inside a limited equivalent region. The simulations demonstrate that relative errors as low as 1-2.5\% are achievable based on the specifics of the source and shape of the open surface.
\end{abstract}

\section{INTRODUCTION}

The equivalence principle has long been used to replace the original sources by suitable equivalent source distributions satisfying the boundary conditions [1]. Since this theorem is based on the uniqueness theorem, the degree of accuracy directly depends on the knowledge of the tangential components of the incident fields over an imaginary or physical closed surface of interest.

We establish a method able to regenerate the original fields inside a limited region near or around an open surface. Considering that equivalence theorems are postulated based on closed surfaces, it is intuitively reasonable to define an equivalent state using a closed surface, and then proceed by transforming the closed surface to an open one by introducing the new method, "windowed equivalence principle" (WEP).

Fig. 1(a) illustrates the original sources that completely radiate the fields $\boldsymbol{E}$ and $\boldsymbol{H}$. The volume within $S$, the region of interest, is denoted by $V_{\mathrm{i}}$, and outside of $S$ by $V_{\mathrm{o}}$. Fig. 1(b) defines an equivalent problem containing the original fields $\boldsymbol{E}$, $\boldsymbol{H}$ of $V_{\mathrm{i}}$, and zero fields outside of $S$. For these fields to exist inside and outside of $S$, there must exist equivalent surface current densities $\boldsymbol{J}_{s}=\boldsymbol{H} \times \hat{\boldsymbol{n}}$ and $\boldsymbol{M}_{s}=\hat{\boldsymbol{n}} \times \boldsymbol{E}$ over $S$ that satisfy the boundary conditions.

To accurately reproduce the original fields inside $S, \boldsymbol{J}_{s}$ and $M_{s}$ must be computed over the entire surface no matter how large it is. Electrically large problems require a huge amount of computer time and memory to compute and store these equivalent currents. More complications arise when the region of interest is not bounded with a closed surface. Legitimately, we may not use the equivalence principle inside any region with open boundaries; otherwise, unpredictable computational errors are unavoidable. Although such errors exist, one can define a limited area inside the region of interest

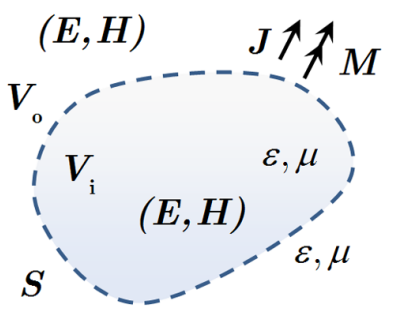

(a) Original problem.

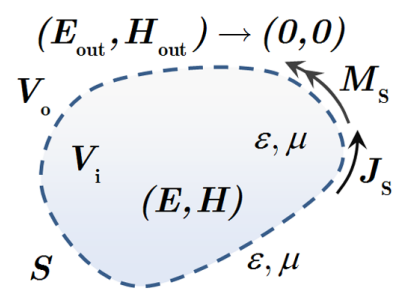

(b) Equivalent problem.
Fig. 1. Surface equivalence theorem.

where the maximum computational error can be kept below a predetermined level. This method is very useful in some practical problems, where the tangential field components are known over the surface but the original sources are not easily accessible and there is a need to compute the original fields inside a limited volume around the open surface.

\section{Proposed METhod}

To reduce the computational field errors imposed by using equivalent surface currents on open surfaces, we propose to incorporate the well-known window functions, which are commonly used in signal-processing applications [2]. Practically, a window function helps smoothen the sudden variations of surface currents due to sharp truncations at the boundaries, mainly by tapering down the amplitude of surface currents over the effective domain of the function. Based on our numerical experiences, using a generalized Hamming window function defined by $W_{\text {ham }}(\rho)=\alpha-\beta \cos \left(\frac{\pi \rho}{R}\right)$ [3] and carefully choosing the coefficients as $\alpha=-\beta=1 / 2$, and shifting the effective domain, i.e., $R$ to an arbitrary range of $\left(R_{\text {mid }} \mapsto R\right)$, gives us the shifted version of a one-dimensional (1-D) Hanning window:

$$
W_{\mathrm{han}}^{1 \mathrm{D}}(\rho)=\frac{1}{2}+\frac{1}{2} \cos \left(\pi \frac{\rho-R_{\mathrm{mid}}}{R-R_{\mathrm{mid}}}\right) .
$$

This type of windowing predominantly alleviates truncation errors for those surfaces with a circular symmetry, as discussed in [4]. But for a rectangular surface, we must consider sharp truncation at the edges, hence, we must transform the circularly symmetric Hanning window function to a centrally symmetric two-dimensional (2-D) version:

$$
W_{\mathrm{han}}^{2 \mathrm{D}}=\left[\frac{1}{2}+\frac{1}{2} \cos \left(\pi \frac{x-a}{A-a}\right)\right] \cdot\left[\frac{1}{2}+\frac{1}{2} \cos \left(\pi \frac{y-b}{B-b}\right)\right],
$$



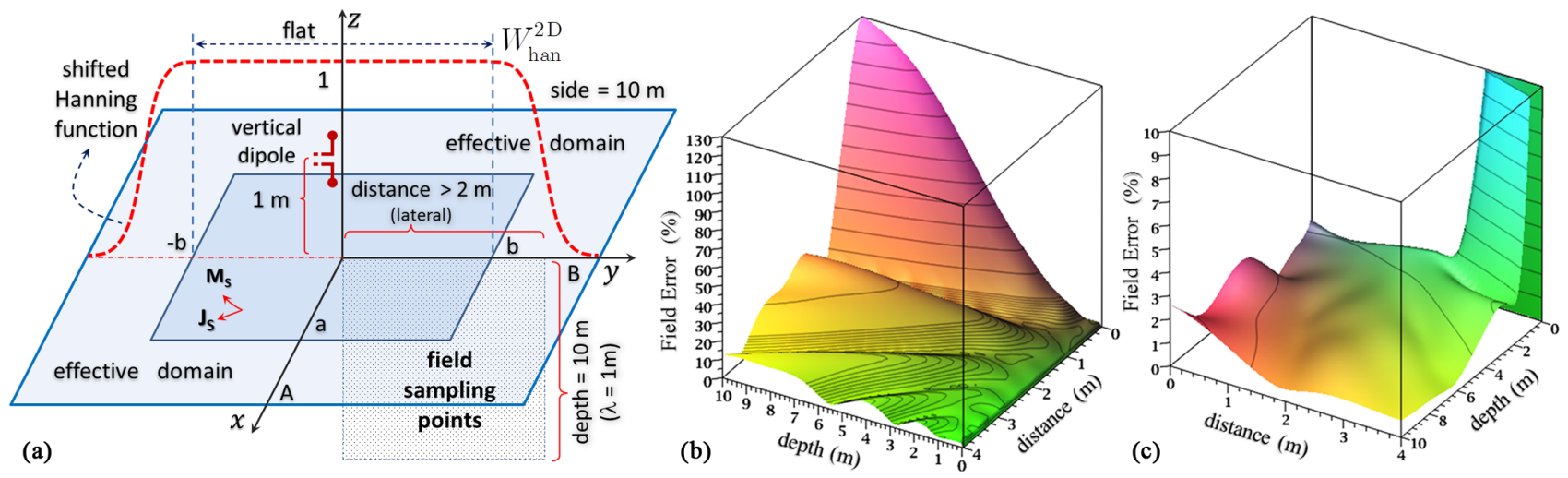

Fig. 2. (a) Important parameters of a 2-D Hanning window as well as the field sampling points underneath an imaginary planar square shape of side $2 A=10 \mathrm{~m}$ illuminated by a vertical dipole positioned $1 \mathrm{~m}$ above the surface. (b) Percentage of field error between computed electric field and incident electric field for a vertical electric dipole without windowing. Contour lines range from 1\% to $10 \%$ and then from $20 \%$ to $120 \%$. (c) The effect of applying a 2-D Hanning window with $R_{\mathrm{pw}}=50 \%$. Contour lines begin at $1 \%$ and continue to $10 \%$.

where $(A, B)$ and $(a, b)$ are defined in Fig. 2(a). Note that only the windowed area between two rectangles specified by $(a, b)$ and $(A, B)$ are progressively affected by this function. Furthermore, we define a parameter, namely, the "partial windowing ratio," as $R_{\mathrm{pw}}=(A-a) / A=(B-b) / B$, which will be used to optimise the numerical results.

We commence by discretizing the surface, and then use the Rao-Wilton-Glisson (RWG) functions [5] to expand $\boldsymbol{J}_{s}$ and $\boldsymbol{M}_{s}$ over the surface. Finally, we apply an appropriate window function to minimize the error. We must take into account other sources of error including computing accuracy, singularity extraction, and discretization, but our first priority is to reduce the computational errors arising from the use of open surfaces.

\section{Numerical Results}

We wish to obtain a conceptual idea regarding two different cases; first, the fields due to using equivalent surface currents over an open surface, and second, what happens when we apply a window function over the same surface. To determine this information, we will use a short vertical dipole [6] as a source. The $300-\mathrm{MHz}$ dipole $(\lambda=1 \mathrm{~m})$ is positioned $1 \mathrm{~m}$ above an imaginary planar square of side $2 A=10 \mathrm{~m}$, as depicted in Fig. 2(a). For numerical purposes, we define a metric, able to show any type of error incurred by truncated currents at the boundaries, with respect to the original incident fields radiated by the dipole, as

$$
\text { Field Error }(\%)=100 \times \frac{\left|\boldsymbol{E}^{\text {computed }}-\boldsymbol{E}^{\text {incident }}\right|}{\left|\boldsymbol{E}^{\text {incident }}\right|} \text {. }
$$

Fig. 2(b) illustrates the percentage of field error as a function of lateral distance from the source and vertical distance (depth) from the surface, both in meters. For easy reading, the contours in the figure are arranged from $1 \%$ to $10 \%$ and then from $20 \%$ to $120 \%$. The figure shows that moving far away from the surface in any direction dramatically increases the amount of error. To reduce this error ( $>120 \%)$, we apply a 2-D Hanning window with $R_{\mathrm{pw}}=50 \%$ on the coefficients obtained from the RWG expansion. The result shown in Fig. 2(c), reveals that inside almost the entire area, the field error is below $2.5 \%$.

Repeating the same procedure for a horizontal dipole yields even smaller errors. A wide range of simulations with different scenarios confirm the validity and advantage of this method. It is noteworthy to mention that all of these observations discussed so far depend on the proper choice of window function and the amount of $R_{\mathrm{pw}}$.

\section{CONCLUSIONS}

We propose a new method, namely, WEP, to effectively decrease computational field error due to using equivalent current sources over open surfaces. Proper choice of the window parameters is mainly based on the specifics of the source and shape of the open surface. Errors below $2.5 \%$ are achievable in relatively large volumes using WEP.

\section{ACKNOWLEDGEMENT}

This work was supported by the Scientific and Technical Research Council of Turkey (TUBITAK) under Research Grant 111E203, by Schlumberger-Doll Research (SDR), and by contracts from ASELSAN, Turkish Aerospace Industries (TAI), and the Undersecretariat for Defense Industries (SSM).

\section{REFERENCES}

[1] S. Schelkunoff, "Some equivalence theorems of electromagnetics and their application to radiation problems," Bell Syst. Tech. J., vol. 15, no. 1, pp. 92-112, 1936.

[2] F. Harris, "On the use of windows for harmonic analysis with the discrete fourier transform," Proc. IEEE, vol. 66, no. 1, pp. 51-83, 1978.

[3] [Online]. Available: http://www.mathworks.com/help/signal/ref/hann.html

[4] M. Takrimi and L. Gürel, "Windowed equivalence principle for open surfaces," in Proc. Computational Electromagnetics Workshop, CEM'13, Izmir, Turkey, August 2013, pp. 23-25.

[5] S. Rao, D. Wilton, and A. Glisson, "Electromagnetic scattering by surfaces of arbitrary shape," IEEE Trans. Antennas Propag., vol. 30, no. 3, pp. 409-418, May 1982.

[6] C. A. Balanis, Antenna Theory: Analysis and Design. John Wiley \& Sons, 2012. 